\title{
Direct Mass Limits for Chiral Fourth-Generation Quarks in All Mixing Scenarios
}

\author{
Christian J. Flacco, ${ }^{1, *}$ Daniel Whiteson,,${ }^{1} \dagger$ Tim M.P. Tait,${ }^{1,}{ }^{\ddagger}$ and Shaouly Bar-Shalom ${ }^{2}, \S$ \\ ${ }^{1}$ Department of Physics and Astronomy, University of California, Irvine, CA 92697, USA \\ ${ }^{2}$ Physics Department, Technion-Institute of Technology, Haifa 32000, Israel
}

\begin{abstract}
Present limits on chiral fourth-generation quark masses $m_{b^{\prime}}$ and $m_{t^{\prime}}$ are broadly generalized and strengthened by combining both $t^{\prime}$ and $b^{\prime}$ decays and considering the full range of $t^{\prime}$ and $b^{\prime}$ flavormixing scenarios (with the lighter generations). Various characteristic mass-splitting choices are considered. With $m_{t^{\prime}}>m_{b^{\prime}}$ we find that CDF limits on the $b^{\prime}$ mass vary by no more than $10-20 \%$ with any choice of flavor-mixing, while for the $t^{\prime}$ mass, we typically find stronger bounds, in some cases up to $m_{t^{\prime}}>430 \mathrm{GeV}$. For $m_{b^{\prime}}>m_{t^{\prime}}$ we find $m_{b^{\prime}}>380-430 \mathrm{GeV}$, depending on the flavor-mixing and the size of the $m_{t^{\prime}}-m_{b^{\prime}}$ mass splitting.
\end{abstract}

PACS numbers:

Despite many constraints by precision electroweak data, the number of simple Standard Model (SM) quark generations has not been definitively established. Recent searches by the CDF Collaboration placing lower limits on the masses of such objects $[1,2]$ have been construed to leave open significant portions of the theoretical landscape that include a light fourth generation [3-7]. In this letter, we offer a treatment of the published data that fully probes the robustness of these limits and applies them to heavy quark decay modes for which there are no present direct limits. By considering all scenarios of mixing among four SM generations, we demonstrate that present data exclude a chiral fourth generation quark with a mass up to nearly $300 \mathrm{GeV}$, independent of the flavor-mixing assumptions. In so doing we establish a methodology for interpretation of future mass limits.

Recent searches for direct production of fourth generation quarks, denoted $t^{\prime}$ and $b^{\prime}$ for the up- and down-type, found $m_{t^{\prime}}>335 \mathrm{GeV}$ [1] and $m_{b^{\prime}}>338 \mathrm{GeV}$ [2], assuming $\mathcal{B}\left(t^{\prime} \rightarrow W\{q=d, s, b\}\right)=100 \%$ and $\mathcal{B}\left(b^{\prime} \rightarrow W t\right)=$ $100 \%$ respectively. Each assumes that an individual contribution from one flavor of fourth-generation quark comprises the entire signal. These searches have been interpreted under the assumptions of $m_{t^{\prime}}-m_{b^{\prime}}<M_{W}$ and negligible mixing of the $\left(t^{\prime}, b^{\prime}\right)$ states with the two lightest quark generations. To account for electroweak precision data [8] and flavor data [9] such conditions are typically required for SM extensions with four quark generations and one Higgs doublet; however, more exotic scenarios have also been proposed $[5-7,10]$.

This subtle theoretical landscape demands a broader view: there is no uniquely interesting set of assumptions under which experimental data should be interpreted. From the experimental view point, choosing a simple set of assumptions allows straightforward, if narrow, interpretation of results. Yet these interpretations may be

\footnotetext{
*Electronic address: cflacco@mac.com

${ }^{\dagger}$ Electronic address: daniel@uci.edu

¥Electronic address: tmptait@gmail.com

$\S$ Electronic address: shaouly@physics.technion.ac.il
}

extended beyond the narrow mixing assumptions, and even applied to previously unexplored decay modes. In this article we consider the possible 4th generation mass spectrum and flavor-mixing space broadly to show that data from the CDF searches set direct limits for arbitrary mixing values and for both cases of $\left|m_{t^{\prime}}-m_{b^{\prime}}\right|>M_{W}$. In particular, we present results for the cases $m_{t^{\prime}}>m_{b^{\prime}}$ and $m_{b^{\prime}}>m_{t^{\prime}}$, and both modest $(50 \mathrm{GeV})$ and large $(100 \mathrm{GeV})$ mass splitting.

CDF analyzed two independent samples: (1) a collection of events containing a single lepton and at least four jets, denoted as the $\ell+4 j$ sample [1], and (2) a collection of events containing two same-charge leptons, two jets (one with a flavor tag) and evidence of neutrinos (missing transverse energy), denoted as the $\ell^{ \pm} \ell^{ \pm} j b E_{T}$ sample [2]. We describe them here.

In $4.6 \mathrm{fb}^{-1}$ of data, CDF searched for $t^{\prime} \rightarrow W\{q=$ $d, s, b\}$ decays in the mode

$$
p \bar{p} \rightarrow t^{\prime} \bar{t}^{\prime} \rightarrow(W \rightarrow l \nu) q\left(W \rightarrow q q^{\prime}\right) q
$$

by requiring a single lepton and at least four jets [1]. The data were analyzed by reconstructing the invariant mass of the candidate $t^{\prime}$ and measuring the total energy in the event. The event selection used the four jets of highest transverse energy in the event, but did not require a flavor signature ( $b$-tag) on any of the jets, making it generally sensitive to $t^{\prime} \rightarrow W\{q=d, s, b\}$. Assuming $\mathcal{B}\left(t^{\prime} \rightarrow W\{q=d, s, b\}\right)=100 \%, \mathrm{CDF}$ found $m_{t}^{\prime}>335$ $\mathrm{GeV}[1]$.

The mass reconstruction used minimum-likelihood fitting methods that depend upon the particular spectrum of final state components. If, for example, one half of an event decayed as $t^{\prime} \rightarrow W\left(b^{\prime} \rightarrow W q\right)$, giving a $W W q W q$ topology, it might satisfy the $\ell+4 j$ selection criteria, but the reconstructed mass distribution for such events would be significantly modified by the additional $W$. Thus, the results cannot be trivially applied to topologies other than $W q W q$. We therefore apply the $\ell+4 j$ results exclusively to $W q W q$ processes.

CDF also searched for $b^{\prime} \rightarrow W t$ decays in $2.7 \mathrm{fb}^{-1}$ of data, in the same-charge lepton mode:

$$
p \bar{p} \rightarrow b^{\prime} \bar{b}^{\prime} \rightarrow W t W \bar{t} \rightarrow W W b W W \bar{b}
$$




$$
\rightarrow\left(\ell^{ \pm} \nu\right)\left(q q^{\prime}\right) b\left(q q^{\prime}\right)\left(\ell^{ \pm} \nu\right) \bar{b}
$$

by requiring two same-charge leptons, at least two jets (at least one with a $b$-tag), and missing transverse energy of at least $20 \mathrm{GeV}$ [2]. Given the small backgrounds, multiple neutrinos and large jet multiplicity in the sample, CDF did not reconstruct the $b^{\prime}$ mass, but instead fit the observed jet multiplicity to signal and background templates generated from simulations. Assuming $\mathcal{B}\left(b^{\prime} \rightarrow W t\right)=100 \%$, CDF found $m_{b}^{\prime}>338 \mathrm{GeV}$ [2].

The $\ell^{ \pm} \ell^{ \pm} j b E_{T}$ analysis did not use final-state dependent fits, thus results are process-independent and may be applied to any process producing the $\ell^{ \pm} \ell^{ \pm} j b E_{T}$ signal. For example, $t^{\prime} \rightarrow W b^{\prime} \rightarrow W W t \rightarrow W W W b$ decays would produce a six- $W$, two- $b$ signature, with higher jet multiplicity and larger acceptance to the $\ell^{ \pm} \ell^{ \pm} j b E_{T}$ sample than the simple four- $W$, two- $b$ signature. In this analysis, we therefore apply the $\ell^{ \pm} \ell^{ \pm} j b E_{T}$ results inclusively to processes resulting in at least four $W$ bosons and two $b$ quarks.

The $\ell+4 j$ and $\ell^{ \pm} \ell^{ \pm} j b E_{T}$ data samples are complementary. In the case that the fourth generation quarks decay exclusively at tree level through the charged current electroweak interaction (assured for a chiral fourth generation), the two searches can be minimally understood to probe two corners of a two-dimensional interval in branching fraction space. In particular, for the case where the $t^{\prime}$ is heavier than the $b^{\prime}$ the topologies of $b^{\prime}$ and $t^{\prime}$ decays are determined by four branching fractions, two of which are independent:

$$
\begin{aligned}
\mathcal{B}\left(t^{\prime} \rightarrow W b^{\prime}\right) & =1-\mathcal{B}\left(t^{\prime} \rightarrow W\{q=d, s, b\}\right) \\
\mathcal{B}\left(b^{\prime} \rightarrow W t\right) & =1-\mathcal{B}\left(b^{\prime} \rightarrow W\{q=u, c\}\right)
\end{aligned}
$$

as shown in Fig. 1. In this representation, the $\ell^{ \pm} \ell^{ \pm} j b E_{T}$ and $\ell+4 j$ analyses probe complementary regions (see Fig. 1).

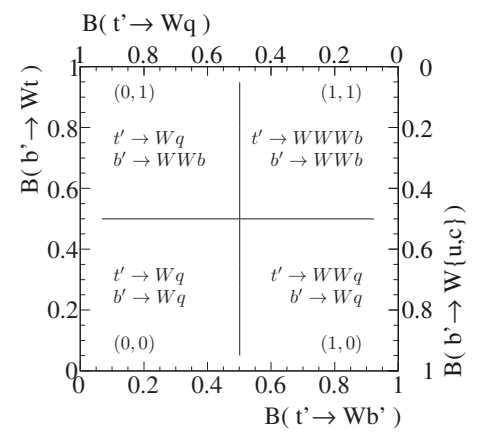

FIG. 1: The flavor-mixing intervals overlaid with a table of the processes contributing to the axis vertices.

We consider the implications of the CDF data to various two-flavor $\left(t^{\prime}\right.$ and $\left.b^{\prime}\right)$ scenarios, characterized by the $t^{\prime}-b^{\prime}$ mass splitting and flavor-mixing rates. To extend the interpretations of the published results, we use the relationship among event yield, cross section and acceptance to interpret the observed yield limits under the
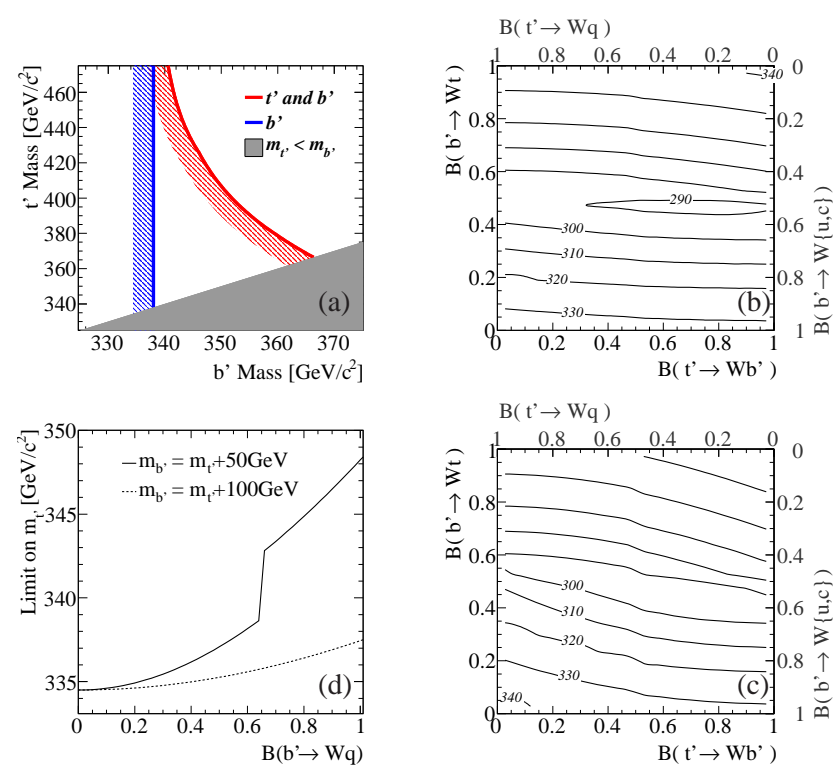

FIG. 2: (a) Limits for the combined hypothesis $t^{\prime} \rightarrow W b^{\prime}$ and $b^{\prime} \rightarrow W t$ derived from the $\ell^{ \pm} \ell^{ \pm} j b E_{T}$ data. Also plotted is the previous CDF limit for the individual $b^{\prime}$ case. (b) Limits on $b^{\prime}$ mass from the combined $\ell^{ \pm} \ell^{ \pm} j b E_{T}$ and $\ell+4 j$ data, as a function of branching fractions $\mathcal{B}\left(t^{\prime} \rightarrow W b^{\prime}\right)=1-\mathcal{B}\left(t^{\prime} \rightarrow\right.$ $W q)[q=d, s, b]$ and $\mathcal{B}\left(b^{\prime} \rightarrow W t\right)=1-\mathcal{B}\left(b^{\prime} \rightarrow W q\right)[q=u, c]$ for the case $m_{t^{\prime}}=m_{b^{\prime}}+100 \mathrm{GeV}$ (c) Same as in (b) but for $m_{t^{\prime}}=m_{b^{\prime}}+50 \mathrm{GeV}$. (d) Limits on $t^{\prime}$ mass from $\ell+4 j$ data as a function of $\operatorname{BR}\left(b^{\prime} \rightarrow W\{q=u, c\}\right)$, in the inverted mass splitting case $m_{b^{\prime}}>m_{t^{\prime}}$.

varying assumptions. This requires parametrization of the variation in relative acceptance due to modification of the signal-source model.

First, we consider the $\ell^{ \pm} \ell^{ \pm} j b E_{T}$ sample, interpreted under the mass splitting assumption $m_{t^{\prime}}>m_{b^{\prime}}$. In the original $\ell^{ \pm} \ell^{ \pm} j b E_{T}$ analysis, the event yield was assumed to come from an individual $b^{\prime}$. Here, we interpret this yield under a model with both $t^{\prime}$ and $b^{\prime}$ contributions, where the decay modes of interest are

$$
\begin{aligned}
& b^{\prime} \rightarrow W t \rightarrow W W b \\
& t^{\prime} \rightarrow W b^{\prime} \rightarrow W W t \rightarrow W W W b
\end{aligned}
$$

which corresponds to the boundary case $(1,1)$ in branching fraction space. The $t^{\prime} \rightarrow W W W b$ mode has no prior direct limit despite having a similar signature with larger acceptance in the $\ell^{ \pm} \ell^{ \pm} j b E_{T}$ dataset due to the two additional $W \mathrm{~s}$ in the intermediate decay chain. Indeed, if both fourth-generation quarks exist, we expect to select both modes in the $\ell^{ \pm} \ell^{ \pm} j b E_{T}$ sample.

In general, the ratio of event yield to the integrated luminosity $N / L$ equals the cross section multiplied by the acceptance rate. For a particular process, such as an individual $b^{\prime}$, this gives the limit on cross section: $\sigma_{b^{\prime}}=\frac{N}{L \cdot \epsilon_{b^{\prime}}}$ where $\epsilon_{b^{\prime}}$ is the acceptance rate for the observed process within the experimental selection constraints. However, we can also consider the case with two contributions (i.e., 
from $t^{\prime}$ and $b^{\prime}$ ) if we know the relative acceptance rates between the corresponding processes, $\epsilon_{r e l}$, and if the two cross sections are dependent:

$$
\frac{N}{\left(L \cdot \epsilon_{b^{\prime}}\right)}=\sigma_{b^{\prime}}+\epsilon_{r e l} \cdot \sigma_{t^{\prime}}\left(\sigma_{b^{\prime}}\right) .
$$

The $t^{\prime}$ and $b^{\prime}$ cross sections, nearly the same up to the $t^{\prime}-b^{\prime}$ mass difference, are both determined by perturbative QCD. (Electroweak production modes of pairs of the fourth generation quarks are expected to be $\leq 1 \%$ at the Tevatron). This is parameterized from the next-to-leading order cross-section calculations for strong force production of massive quarks [11], by fitting the published theoretical cross-section to an exponential model in the local region of mass under consideration: $\sigma_{t^{\prime}}=\sigma_{b^{\prime}} \exp -\frac{m_{t^{\prime}}-m_{b^{\prime}}}{M}$, where $M$ is found to be $40 \mathrm{GeV}$.

This relationship yields cross-section limits and, by extension, mass limits on $b^{\prime}$ for a spectrum of assumed $t^{\prime}$ masses. The relative efficiency $\epsilon_{\text {rel }}$ between the original model and the model explicitly including both $t^{\prime}$ and $b^{\prime}$ was estimated using simulated data. The model for $\epsilon_{\text {rel }}$ accounts for increased acceptance of $t^{\prime}$ as the fourthgeneration mass difference increases, with a plateau beyond $m_{W}$. This method was validated by varying the model for $\epsilon_{r e l}$. The resulting mass limits were found to be stable. We find that the additional contribution from the $t^{\prime} \rightarrow W b^{\prime}$ decay gives the limits $m_{b^{\prime}}>340-360$ depending on $m_{t^{\prime}}$ (Fig. 2a), stronger than the previous CDF individual $b^{\prime}$ limit.

We probe the full two-dimensional branching fraction interval by calculating the dependence of the event yield on the branching fractions explicitly. As the branching fractions to the reconstructed states vary, the acceptances and reconstruction efficiencies of the processes of interest vary accordingly. Considering these effects, we calculate the acceptances of $b^{\prime}$ and $t^{\prime}$ in both the $\ell+4 j$ and $\ell^{ \pm} \ell^{ \pm} j b E_{T}$ samples.

The expected signal yield for one process relative to another is proportional to the relative production rates and final-state reconstruction efficiencies. The relative signal production rates have two factors: the relative cross section for initial state production (i.e., $t^{\prime} \bar{t}^{\prime}$ and $\left.b^{\prime} \bar{b}^{\prime}\right)$ and the branching ratios of the involved processes. For several processes contributing to a signal there are multiple terms of this form. With the event yield fixed at its observed value, we isolate the cross section, and express it in terms of the previously measured limit and an effective relative acceptance. The effective relative acceptance $(A)$ includes acceptance terms for all processes considered, each scaled by the relative cross-section.

For the $\ell+4 j$ sample considered with the classical splitting case $m_{t^{\prime}}>m_{b^{\prime}}$, there are no relative reconstruction efficiencies to consider so the expression for the relative expected yields as a function of $\beta_{b^{\prime}}=\mathcal{B}\left(b^{\prime} \rightarrow W t\right)$ and $\beta_{t^{\prime}}=\mathcal{B}\left(t^{\prime} \rightarrow W b^{\prime}\right)$ is fairly simple:

$$
A\left(\beta_{b^{\prime}}, \beta_{t^{\prime}}\right)=\left(1-\beta_{b^{\prime}}\right)^{2}+\sigma_{\text {rel }}\left(1-\beta_{t^{\prime}}\right)^{2},
$$

where $\sigma_{r e l} \equiv \sigma_{t}^{\prime} / \sigma_{b}^{\prime}$. This treatment produces limits on the mass of the $b^{\prime}$ as a function of fourth-generation branching fractions.

The $\ell^{ \pm} \ell^{ \pm} j b E_{T}$ case is somewhat more complicated. In addition to $b^{\prime}$, there are two significant $t^{\prime}$ processes that produce the signal selected for the sample analyzed:

$$
\begin{aligned}
t^{\prime} & \rightarrow W b^{\prime} \rightarrow W W t \rightarrow W W W b, \\
t^{\prime} & \rightarrow W b^{\prime} \rightarrow W W c .
\end{aligned}
$$

Jet multiplicity and the flavor-tag requirement imply different relative reconstruction efficiencies for each contribution. These factors are denoted $\epsilon_{N W}$, where $N$ is the number of intermediate $W$-bosons in the process described. They are estimated from simulated data and are calculated relative to the four- $W$ case considered in the original analysis. The effective relative acceptance in this case has the form

$$
\begin{gathered}
A\left(\beta_{b^{\prime}}, \beta_{t^{\prime}}\right)=\beta_{b^{\prime}}^{2}+\beta_{t^{\prime}}^{2} \frac{\sigma_{r e l}}{\epsilon_{b b}}\left[\left(1-\beta_{b^{\prime}}\right)^{2} \epsilon_{c c}\right. \\
\left.+2 \beta_{b^{\prime}}\left(1-\beta_{b^{\prime}}\right) \epsilon_{5 W} \epsilon_{c b}+\beta_{b^{\prime}}^{2} \epsilon_{6 W} \epsilon_{b b}\right],
\end{gathered}
$$

where the individual efficiencies for each combination of decay modes are estimated by producing pairs of massive quarks at tree level using the MadEvent software package [12] for a user-defined model containing $t^{\prime}$ and $b^{\prime}$ and allowing for $\mathcal{O} \sim 1 \%$ mixing with either the third or second generations (i.e., $V_{4 j} \sim \mathcal{O}(0.1)$ for $j=2,3$ ). Under these assumptions, all of the inclusive decay widths (including $t^{\prime} \rightarrow W b^{\prime}$ which is unsuppressed by mixing) are well within the expected jet energy resolution at a hadron collider. The events are generated at $b^{\prime}$ masses of $400 \mathrm{GeV}$ and for two different choices of mass splitting, $50 \mathrm{GeV}$ and $100 \mathrm{GeV}$, representing both on-shell and offshell $W$ bosons in the $t^{\prime} \rightarrow b^{\prime}$ decay. The events are processed into the desired decays modes using the BRIDGE program [13]. As expected, in the region of interest the dependence of efficiencies on quark-mass choices is small.

Terms from the $t^{\prime}$ contribution have unique reconstruction efficiencies due to jet-flavor tagging, expressed in eq. 3 by $\epsilon_{f_{1} f_{2}}$, where $f_{1} f_{2}$ is the flavor combination of the quarks in the final state of the (quark-level) $t^{\prime}$ decay. These efficiencies are given by statistics from the raw efficiencies of the jet-flavor tag to select the beauty (60\%) or charm $(15 \%)$ flavor in a jet:

$$
\begin{aligned}
\epsilon_{b b} & =1-\left(1-\epsilon_{b}\right)^{2} \\
\epsilon_{c b} & =\epsilon_{c}\left(1-\epsilon_{b}\right)+\epsilon_{b}\left(1-\epsilon_{c}\right)+\epsilon_{b} \epsilon_{c} \\
\epsilon_{c c} & =1-\left(1-\epsilon_{c}\right)^{2} .
\end{aligned}
$$

Using the above procedure, we produce limits on the mass of the $b^{\prime}$ as a function of fourth-generation branching fractions.

The two sets of results reveal the complementary sensitivities of the CDF analyses over the interval. However, they are not orthogonal and thus cannot be statistically combined. Instead, the best limits are found by choosing the stronger of two limits at each point in the branching fraction interval. The combined results are presented for two characteristic choices of mass splitting in Fig. 2b,c. 
Finally, we consider the case of an inverted splitting, $m_{b^{\prime}}>m_{t^{\prime}}$. The CDF $\ell+4 j$ data sets a limit on $t^{\prime}$ for every choice of branching fraction in this case, as its assumption that $\mathcal{B}\left(t^{\prime} \rightarrow W q=d, s, b\right)=100 \%$ is always satisfied. If $\mathcal{B}\left(b^{\prime} \rightarrow W q=u, c\right)$ is significant it also contributes, but at a smaller cross-section due to its larger mass. With the roles of $b^{\prime}$ and $t^{\prime}$ reversed, $\sigma_{r e l}$ is inverted accordingly in this case. The relative acceptance as a function of $\beta_{b^{\prime}}=\mathcal{B}\left(b^{\prime} \rightarrow W t^{\prime}\right)$ is given by:

$$
A\left(\beta_{b^{\prime}}\right)=1+\sigma_{\text {rel }} \beta_{b^{\prime 2}} .
$$

On the other hand The $\ell^{ \pm} \ell^{ \pm} j b E_{T}$ sample, sensitive to $b$-type quarks, cannot at its present size set limits in the inverted splitting case, as the limits it produces would imply a lighter $t^{\prime}$ already excluded by the $\ell+4 j$ sample.

Our limits for the inverted case $m_{b^{\prime}}>m_{t^{\prime}}$ with the $\ell+4 j$ sample are shown in Fig. 2d. The displayed discontinuity in the upper curve of Fig. 2d is caused by the corresponding slope in the $\ell+4 j$ result.

To summarize, by analyzing the expectations for relative event yields at CDF under the combined $t^{\prime}, b^{\prime}$ hypothesis, and assuming a continuum of $t^{\prime}$ and $b^{\prime}$ branching fractions, we find that the CDF data imply limits on $m_{b^{\prime}}$ and $m_{t^{\prime}}$ of $290 \mathrm{GeV}$ and greater over the full range of mixing scenarios, for two characteristic choices of the $t^{\prime}-b^{\prime}$ mass splitting: $m_{t^{\prime}}>m_{b^{\prime}}$ and $m_{b^{\prime}}>m_{t^{\prime}}$. The inclusion of a $t^{\prime}$ strengthens the previously obtained $b^{\prime}$ mass limit from the $\ell^{ \pm} \ell^{ \pm} j b E_{T}$ sample; in the $m_{t^{\prime}}>m_{b^{\prime}}$ case, by up to $10 \%$ when $0<m_{t^{\prime}}-m_{b^{\prime}}<M_{W}$.

If a fourth generation of fermions is embedded in theories beyond the SM, then the large splitting case $\left(m_{t^{\prime}}-m_{b^{\prime}}>M_{W}\right)$ and the inverted scenario $\left(m_{b^{\prime}}>m_{t^{\prime}}\right)$ may not be excluded. Indeed, an example given recently in [7] shows that precision EW data can accommodate $m_{t^{\prime}}-m_{b^{\prime}}>M_{W}$ if there are two Higgs doublets. In fact, the compositeness picture emerging from the addition of new heavy fermionic degrees of freedom is more naturally described at low energies by multi-Higgs theories [5-7], for which constraints on the fourth generation parameter space are known to be relaxed [10]. In such cases (i.e., a large $t^{\prime}-b^{\prime}$ mass splitting and/or an inverted splitting), we find new stronger bounds on $m_{b^{\prime}}$ and $m_{t^{\prime}}$, in some mixing scenarios reaching up to $430 \mathrm{GeV}$.

Acknowledgments: The work of CF and DW is supported in part by the Dept. of Energy and the Alfred P. Sloan Foundation. SBS acknowledge research support from the Technion. TT is grateful to the SLAC theory group for their hospitality during which part of this work was completed.
[1] A. Lister arXiv:0810.3349 [hep-ex]; J. Conway et al., CDF/PUB/TOP/PUBLIC/10110.

[2] T. Aaltonen et al. , arXiv:0912.1057 [hep-ex].

[3] P.H. Frampton, P.Q. Hung and M. Sher, Phys. Rept. 330, 263 (2000); B. Holdom, W.S. Hou, T. Hurth, M.L. Mangano, S. Sultansoy, G. Unel, PMC Phys. A3, 4 (2009).

[4] B. Holdom, Phys. Rev. Lett. 57, 2496 (1986) [Erratumibid. 58, 177 (1987); W.A. Bardeen, C.T. Hill and M. Lindner, Phys. Rev. D41, 1647 (1990); C. Hill, M. Luty and E.A. Paschos, Phys. Rev. D43, 3011 (1991); P.Q. Hung and G. Isidori Phys. Lett. B402, 122 (1997).

[5] P.Q. Hung and Chi Xiong, arXiv:0911.3890 [hep-ph]; ibid. arXiv:0911.3892 [hep-ph]; H. J. He, C. T. Hill and T. M. P. Tait, Phys. Rev. D 65, 055006 (2002) [arXiv:hep-ph/0108041].

[6] M. Hashimoto, V.A. Miransky, arXiv:0912.4453 [hep-ph].

[7] M. Hashimoto, arXiv:1001.4335 [hep-ph].

[8] G.D. Kribs, T. Plehn, M. Spannowsky, T.M.P. Tait, Phys. Rev. D76, 075016 (2007); ibid. Nucl. Phys. Proc. Suppl. 177-178, 241-245 (2008); M.S. Chanowitz, Phys. Rev. D79, 113008 (2009); V.A. Novikov, A.N. Rozanov and M.I. Vysotsky, arXiv:0904.4570 [hep-ph] and references therein; J. Erler and P. Langacker, arXiv:1003.3211 [hep-ph].

[9] A. Arhib and W.S. Hou, JHEP 0607, 009 (2006); M. Bobrowski, A. Lenz, J. Riedl and J. Rohrwild, Phys. Rev. D79, 113006 (2009); A. Soni, A.K. Alok, A. Giri, R. Mohanta, S. Nandi, arXiv:0807.1971 [hep-ph]; G. Eilam, B. Melic and J. Trampetic, Phys. Rev. D80, 116003 (2009); A. Soni, A.K. Alok, A. Giri, R. Mohanta, S. Nandi, arXiv:1002.0595 [hep-ph]; A.J. Buras et al., arXiv:1002.2126 [hep-ph].

[10] H. J. He, N. Polonsky and S. f. Su, Phys. Rev. D 64, 053004 (2001) [arXiv:hep-ph/0102144].

[11] R. Bonciani, S. Catani, M. L. Mangano, and P. Nason, Nucl. Phys. B529, 424 (1998); M. Cacciari, S. Frixione, M. L. Mangano, P. Nason, and G. Ridolfi, J. High Energy Phys. 04 (2004) 068.

[12] J. Alwall et al., JHEP 0709, 028 (2007) [arXiv:0706.2334 [hep-ph]].

[13] P. Meade and M. Reece, arXiv:hep-ph/0703031. 